\title{
A Universal Color Image Quality Metric
}

\author{
Alexander Toet ${ }^{*}$ \\ Marcel P. Lucassen \\ TNO Human Factors, Kampweg 5, 3769 DE Soesterberg, The Netherlands
}

\begin{abstract}
We extend a recently introduced universal grayscale image quality index to a newly developed perceptually decorrelated color space. The resulting color image quality index quantifies the distortion of a processed color image relative to its original version. We evaluated the new color image quality metric through observer experiments in which subjects ranked images according to perceived distortion. The metric correlates strongly with human perception and can therefore be used to assess the performance of color image coding and compression schemes, color image enhancement algorithms, synthetic color image generators, and color image fusion schemes.
\end{abstract}

Keywords: image quality, color fidelity

\section{INTRODUCTION}

In many areas of research and development that are concerned with digital imagery there is a real need for a digital metric that quantifies how distorted a processed color image appears relative to the original version of the same image, as perceived by a human observer. For instance, in computer graphics, we may wish to compare the degree of photorealism of two different rendering methods, or to evaluate visible errors between a synthetic and a real scene. In image coding, we may need to evaluate the results of two different compression methods. In image processing, we may need to assess the performance of a new color image enhancement technique. In false-color multispectral image fusion, we may need to quantify the degree of photorealism of fused imagery. Unfortunately, common metrics like the root mean square error (RMSE) are not viable for these tasks because the human visual system does not compare images this way (e.g. ${ }^{1}$ ). Psychophysical evaluation of different image processing techniques is tedious, expensive, and difficult to automate. Hence there is a great need for a validated computational image quality metric that correlates with human perception and that can be used for automatic optimization of the parameters involved in different image processing and rendering techniques.

Over the years a large number of objective metrics have been proposed to assess image and video quality ${ }^{2-29}$. For an overview of different metrics see ${ }^{16,30}$. Ironically, the metrics that are most widely applied today, like the root mean squared error (RMSE) or the peak signal-to-noise ratio (PSNR), are also the ones that correlate least with human perception ${ }^{31-41}$. Human visual system models are more successful, but they are not widely used, since they generally are computationally complex.

Most of the digital image distortion metrics in the literature apply to grayscale images. Only a few studies address color image quality metrics ${ }^{13,17,25,26,42-44}$. Grayscale image quality metrics can in principle be generalized to color image quality metrics by applying them to the three (RGB) color channels individually, and then weighing and combining the errors in the different channels together. However, this direct approach does not relate to human perception. The RGB representation is based on primary relative color. This space does not represent color as perceived and analyzed by the human visual system. The human visual system uses three paths to analyze color images: one for achromatic information and two for chromatic contrast signals. As a result, the individual channels of an RGB color image are perceptually highly correlated. Hence, the RGB image should first be transformed into a perceptually uncorrelated color space before further analysis is performed.

\footnotetext{
${ }^{*}$ E-mail: toet@tm.tno.nl
} 
Most of the algorithms used the literature to quantify the distortion of a processed grayscale image relative to its original version are designed for some special types of distortions, such as blocking artifacts ${ }^{3,4,29}$. Wang and Bovik ${ }^{45}$ recently $^{2}$ introduced a general grayscale image quality metric that quantifies a whole range of local image distortions. The quality index is computationally simple and correlates with the subjective evaluations of human observers for a wide variety of distortions. Here we show that this metric can be extended to color imagery, by applying it to the individual channels of a color image in the newly introduced perceptually decorrelated $l \alpha \beta$ color space ${ }^{46}$, and by combining the results from the individual channels into a weighted vector mean.

\section{THE GRAYSCALE IMAGE QUALITY METRIC}

Wang and Bovik ${ }^{45}$ recently introduced a universal image quality metric $\mathrm{Q}$ that quantifies the distortion of a processed image relative to its original version. The distortion metric is defined as a combination of three factors: loss of correlation, luminance distortion, and contrast distortion. Let $\mathrm{x}=\left\{x_{i} \mid i=1,2, \cdots, N\right\}$ and $y=\left\{y_{i} \mid i=1,2, \cdots, N\right\}$ be the original and processed image signals respectively. The quality index $\mathrm{Q}$ is then given by:

$$
Q=\frac{\sigma_{x y}}{\sigma_{x} \sigma_{y}} \cdot \frac{2 \bar{x} \bar{y}}{(\bar{x})^{2}+(\bar{y})^{2}} \cdot \frac{2 \sigma_{x} \sigma_{y}}{\sigma_{x}^{2}+\sigma_{y}^{2}}
$$

where

$$
\begin{gathered}
\bar{x}=\frac{1}{N} \sum_{i=1}^{N} x_{i} \quad, \quad \bar{y}=\frac{1}{N} \sum_{i=1}^{N} y_{i} \\
\sigma_{x}^{2}=\frac{1}{N-1} \sum_{i=1}^{N}\left(x_{i}-\bar{x}\right)^{2}, \quad \sigma_{y}^{2}=\frac{1}{N-1} \sum_{i=1}^{N}\left(y_{i}-\bar{y}\right)^{2} \\
\sigma_{x y}=\frac{1}{N-1} \sum_{i=1}^{N}\left(x_{i}-\bar{x}\right)\left(y_{i}-\bar{y}\right)
\end{gathered}
$$

The dynamic range of $\mathrm{Q}$ is $[-1,1]$. The maximal value 1 only occurs when both images are identical, i.e. $y_{i}=x_{i}$ for all $i=1,2, \cdots, N$. The minimal value -1 occurs when $y_{i}=2 \bar{x}-x_{i}$ for all $i=1,2, \cdots, N$. The first component in Equation (1) is the correlation coefficient between $\mathrm{x}$ and $\mathrm{y}$, which measures the degree of linear correlation between both images, and has a dynamic range of [-1,1]. The maximal value 1 is obtained when $y_{i}=a x_{i}+b$ for all $i=1,2, \cdots, N$, where $a$ and $b$ are constants and $a>0$. Even if $\mathrm{x}$ and $\mathrm{y}$ are linearly related, there may still occur relative distortions between them. These are evaluated in the second and third components. The second component measures how close the mean luminance both images are, and ranges between [0,1]. It equals 1 when $\bar{x}=\bar{y}$. Since $\sigma_{x}$ and $\sigma_{y}$ can be regarded as estimates of the contrast of $\mathrm{x}$ and $\mathrm{y}$, the third component measures how similar the contrasts of both images are. It also ranges from [0,1]. The highest value 1 is obtained if and only if $\sigma_{x}=\sigma_{y}$.

In practice we usually want to characterize an entire image using a single overall image quality measure. However, image quality is often spatially variant, meaning that different image regions may have different types of distortions. It is therefore more appropriate to measure statistical properties locally and combine them into a single measure. Following Wang $^{45}$ we therefore compute the image quality index $\mathrm{Q}$ over local image regions using a sliding window approach. Starting from the top-left corner of the image, a sliding window of size $8 \times 8$ moves pixel by pixel horizontally and vertically through all the rows and columns of the image until the bottom-right corner is reached. At the $j^{\text {th }}$ step in this procedure the local quality index $Q_{j}$ is computed over the area of the $8 \times 8$ sliding window. If the total number of steps is equal to $M$, the overall image quality index is given by 


$$
Q=\frac{1}{M} \sum_{j=1}^{M} Q_{j}
$$

\section{THE COLOR IMAGE QUALITY METRIC}

In this section we extend the grayscale quality metric $\mathrm{Q}$ to include color by applying it to the individual dimensions of a perceptually decorrelated color space, and combining the individual components in a (weighted) vector mean. The rationale for this approach is the fact that the human visual system processes the retinal image in three decorrelated visual channels: one luminance channel and two color opponent channels. As a result, luminance and color distortions will contribute independently to perceived image quality, and should therefore be calculated independently before combining them into a single overall perceived image quality metric.

The common RGB image representation is based on primary relative color. This space does not represent color as perceived and analyzed by the human visual system. In RGB space, there is a strong correlation between the individual image channels. For instance, most pixels will have large values for the red and green channels if the blue channel is large. The human visual system encodes the chromatic signals conveyed by the three types of retinal cone photoreceptors in an opponent fashion. This color opponency is often interpreted as an attempt to remove correlations in the signals of different cone types that are introduced by the strong overlap of the cone spectral sensitivities ${ }^{47}$. Ruderman et $a l .{ }^{46}$ recently derived the perceptually decorrelated $l \alpha \beta$ color space from a principal component transform of a large ensemble of hyperspectral images that represents a good cross-section of natural scenes.

In the following sections we first discuss the RGB to $l \alpha \beta$ transform. Then we construct the color quality metric by applying the grayscale metric $\mathrm{Q}$ to each of the channels in the $l \alpha \beta$ color space.

\subsection{The RGB to $l \alpha \beta$ transform}

First the RGB tristimulus values are converted to device independent XYZ tristimulus values. This conversion depends on the characteristics of the display on which the image was originally intended to be displayed. Because that information is rarely available, it is common practice to use a device-independent conversion that maps white in the chromaticity diagram to white in RGB space and vice versa ${ }^{48}$.

$$
\left[\begin{array}{l}
X \\
Y \\
Z
\end{array}\right]=\left[\begin{array}{lll}
0.5141 & 0.3239 & 0.1604 \\
0.2651 & 0.6702 & 0.0641 \\
0.0241 & 0.1228 & 0.8444
\end{array}\right]\left[\begin{array}{l}
R \\
G \\
B
\end{array}\right]
$$

The device independent XYZ values are then converted to LMS space by

$$
\left[\begin{array}{c}
L \\
M \\
S
\end{array}\right]=\left[\begin{array}{rrr}
0.3897 & 0.6890 & -0.0787 \\
-0.2298 & 1.1834 & 0.0464 \\
0.0000 & 0.0000 & 1.0000
\end{array}\right]\left[\begin{array}{c}
X \\
Y \\
Z
\end{array}\right]
$$

Combination of (3) and (4) results in 


$$
\left[\begin{array}{c}
L \\
M \\
S
\end{array}\right]=\left[\begin{array}{lll}
0.3811 & 0.5783 & 0.0402 \\
0.1967 & 0.7244 & 0.0782 \\
0.0241 & 0.1288 & 0.8444
\end{array}\right]\left[\begin{array}{l}
R \\
G \\
B
\end{array}\right]
$$

The data in this color space shows a great deal of skew, which is largely eliminated by taking a logarithmic transform:

$$
\begin{aligned}
\boldsymbol{L} & =\log L \\
\boldsymbol{M} & =\log M \\
\boldsymbol{S} & =\log S
\end{aligned}
$$

The inverse transform from $\boldsymbol{L M S}$ cone space back to RGB space is as follows. First, the $\boldsymbol{L M S}$ pixel values are raised to the power ten to go back to linear LMS space. Then, the data can be converted from LMS to RGB using the inverse transform of Equation (5):

$$
\left[\begin{array}{l}
R \\
G \\
B
\end{array}\right]=\left[\begin{array}{rrr}
4.4679 & -3.5873 & 0.1193 \\
-1.2186 & 2.3809 & -0.1624 \\
0.0497 & -0.2439 & 1.2045
\end{array}\right]\left[\begin{array}{c}
L \\
M \\
S
\end{array}\right]
$$

Ruderman $e . a{ }^{46}$ recently derived a color space, called $l \alpha \beta$, which effectively minimises the correlation between the $\boldsymbol{L M S}$ axes. This result was derived from a principal component transform to the logarithmic $\boldsymbol{L M S}$ cone space representation of a large ensemble of hyperspectral images that represented a good cross-section of natural scenes. The principal axes encode fluctuations along an achromatic direction $(l)$, a yellow-blue opponent direction $(\alpha)$, and a red-green opponent direction $(\beta)$. The resulting data representation is compact and symmetrical, and provides automatic decorrelation to higher than second order.

Ruderman e.a. ${ }^{46}$ presented the following simple transform to decorrelate the axes in the $\boldsymbol{L M S}$ space:

$$
\left[\begin{array}{l}
l \\
\alpha \\
\beta
\end{array}\right]=\left[\begin{array}{ccc}
\frac{1}{\sqrt{3}} & 0 & 0 \\
0 & \frac{1}{\sqrt{6}} & 0 \\
0 & 0 & \frac{1}{\sqrt{2}}
\end{array}\right]\left[\begin{array}{ccc}
1 & 1 & 1 \\
1 & 1 & -2 \\
1 & -1 & 0
\end{array}\right]\left[\begin{array}{c}
\boldsymbol{L} \\
\boldsymbol{M} \\
\boldsymbol{S}
\end{array}\right]
$$

If we think of the $\mathbf{L}$ channel as red, the $\mathbf{M}$ as green, and $\mathbf{S}$ as blue, we see that this is a variant of a color opponent model:

$$
\begin{aligned}
& \text { Achromatic } \propto r+g+b \\
& \text { Yellow-blue } \propto r+g-b \\
& \text { Red-green } \propto r-g
\end{aligned}
$$

The resulting data representation is compact and symmetrical, and provides automatic decorrelation to higher than second order. 


\subsection{Construction of the color quality metric}

The color quality metric $Q_{\text {color }}$ is defined as:

$$
Q_{\text {color }}=\sqrt{w_{l}\left(Q_{l}\right)^{2}+w_{\alpha}\left(Q_{\alpha}\right)^{2}+w_{\beta}\left(Q_{\beta}\right)^{2}}
$$

where $Q_{l}, Q_{\alpha}, Q_{\beta}$ represent respectively the quality factors given by Equation 2, computed for each of the individual $l \alpha \beta$ color channels, and $w_{b}, w_{\alpha}, w_{\beta}$ are the corresponding weights attributed to the perceived distortions in each of these channels. The $\mathrm{Q}$ values corresponding to the image distortions used in this study were always positive. Equation (10) is designed in analogy to most modern color difference equations ${ }^{49}$.

\section{OBSERVER EXPERIMENTS}

To assess the agreement between our color image quality metric and human visual perception we performed observer experiments in which subjects ranked images according to perceived distortion. The distortions were produced by quantizing the original images along each of the individual dimensions in the perceptually decorrelated $l \alpha \beta$ color space. Since there are many nonlinearities in the way the visual system responds to the retinal image, we can not expect a linear relation between the color image quality metric and the perceived amount of distortion. However, since the metric does increase monotonically with the perceived amount of distortion, it induces a ranking according to the amount of distortion. This ranking should correlate with the ranking produced by the human observers.

\subsection{Stimuli}

The two original 24 bits color reference images used in this study are shown in Figure 1. These images were selected because they show a significant amount of spatial detail on different levels of resolution in combination with a large variation in colors. The "Mandrill" image is 512x512 pixels in size, and the "parrots" image is 384x256 pixels in size, each pixel being represented by three bytes (one for each of the R,G, and B channels). For each reference image, a set of degraded images was constructed as follows. First, the original image was transformed into the perceptually decorrelated $l \alpha \beta$ representation. In this space, the individual channels of the reference image were progressively and uniformly quantized. Uniform quantization was performed by dividing the color space range of the original images into a given number of equally large intervals. Coefficients inside an interval were attributed the value of the lower bound of the interval. The set of quantization intervals was successively chosen such that the quality index $Q$ from Equation (2) was approximately evenly distributed between 0.1 and 0.9 . Finally, the resulting quantized images were transformed back to RGB space for display. The effect of quantization along the $l$ dimension is a reduction of the mean luminance of the image. Quantization along the $\alpha$ and $\beta$ dimensions results in an overall color shift.

We used two different image sets. Images in the first set were quantized in a single channel only $(l, \alpha$ or $\beta)$, and in 7 progressive steps. Images in the second set were distorted in two channels simultaneously (either in $l$ and $\alpha, l$ and $\beta$, or $\alpha$ and $\beta$ ), and again in 7 steps. As a result, each of the two original reference images has 21 corresponding degraded versions in both sets (resulting from the 7 quantization steps in respectively each of the three $l \alpha \beta$ channels in the first set, and for each of the three combinations of 2 individual channels in the second set).

We printed color hardcopies of the reference images and their corresponding quantized representations on high quality glossy photographic paper, using a 600 dpi laser printer. The printed images were $7 \times 7 \mathrm{~cm}^{2}$ in size.

\subsection{Subjects}

Subjects were trained observers, men and women between the ages of 18 and 60. All had normal or corrected-to-normal vision, and no known color deficiencies. 


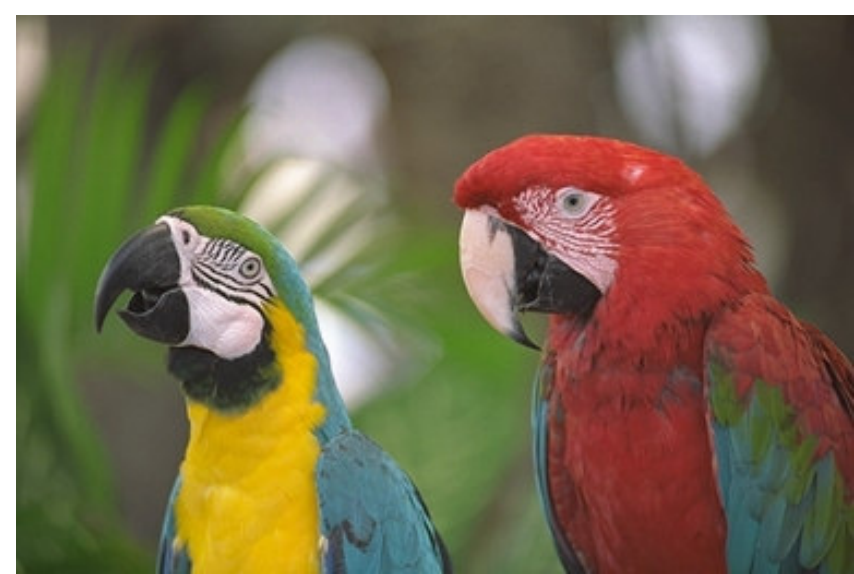

(a)

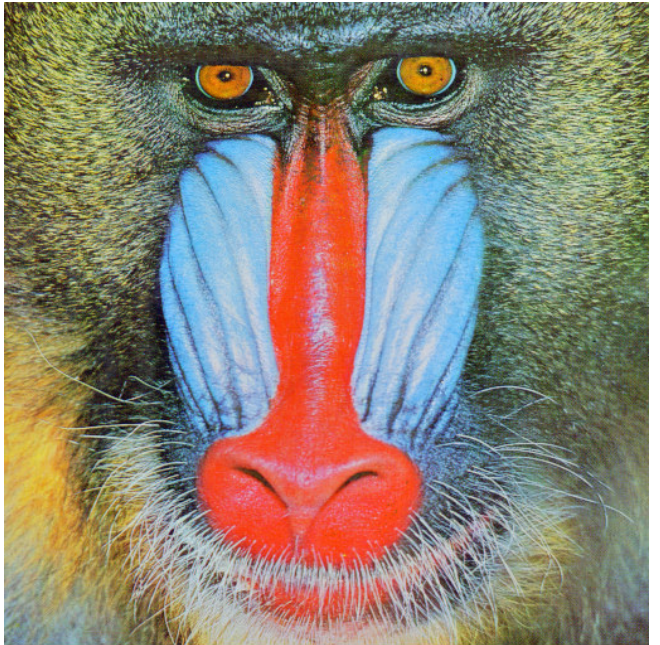

(b)

Figure 1. The original 24 bits RGB color images used in the experiments. The first image (a) represents two parrots, and the second image (b) shows the face of a Mandrill monkey.

\subsection{Experimental design}

We collected rank ordering data from subjects on the sets of printed color images. First, the subjects were given an original (unprocessed) image that served as a reference. Then, on each run, subjects were handed a randomly ordered set of images, corresponding to progressively quantized versions of the reference image. The subjects were asked to rank each series of degraded (quantized) color images by how similar each image was in comparison to the given reference image. The subjects had the ability to change, as required, the classification that they had already done by doing permutations between images until all images were ranked. They had no constraint of time to do this task. We asked the subjects to keep the images on a viewing stand as they sorted them and to keep their viewing distance fixed at approximately $30 \mathrm{~cm}$. At this distance the visual angle of the images is about 4 degrees.

The prints were presented to the observers in a Macbetch SpectraLight II booth, and thus viewed under homogeneous lighting. The luminance reflected from the print was maximally $450 \mathrm{~cd} / \mathrm{m}^{2}$ (white paper). The color temperature of the illuminant is approximately $6430 \mathrm{~K}$.

To measure how close the rankings produced by the human observers agree with the ranking induced by the metric we computed the coefficient $T_{c}$, which corresponds to the correlation between the set of observer rankings and the ranking induced by the metric ${ }^{50}$. $T_{c}$, is the average of the Kendall rank-order correlation coefficients between each ranker and the metric ranking.

We performed three different experiments.

1. In the first experiment, 16 observers ranked 7 images that were distorted in a single color channel only, for each of the color channels separately and consecutively.

2. In the second experiment, 4 subjects ranked a mixed subset of 12 of the images used in the first experiment, such that each set contained 4 progressively quantized versions of the original image for each of the three color channels.

3. In the third experiment, 4 observers ranked 21 images, corresponding to 7 progressively quantized versions of the original image for each of the three color channels. The images were selected such that the quality metric $Q$ was well distributed for each of the individual color channels. 


\subsection{Results}


Figure 2. Results of the three experiments. Top row: Experiment 1. Middle row: Experiment 2. Bottom row: Experiment 3. See text for explanation.

Shown in Figure 3 are the results of three experiments. In the first experiment (top images in Fig. 3), the images were only distorted in a single channel $(l, \alpha$ or $\beta)$ as described in section 4.1. The subjects made rankings for the 7 images in 
each of the three channels separately. Good correlation between the quality metric Q and the average ranking is obtained. In the second experiment (middle images in Fig. 3), we selected 12 images out of the 21 used in experiment 1 so that the quality metric in each channel was well distributed. The subjects made rankings for these 12 images (mixed up). The data indicate that the quality levels of the three channels start to compete. At the same $\mathrm{Q}$ value, distortions in the $l$ channel are given the lowest ranking, followed by the distortions in the $\alpha$ channel and the $\beta$ channel, respectively. Or, in other words, distortions in the $\beta$ channel are less visible than the same amount of distortion in the $\alpha$ channel and the $l$ channel. In the third experiment (bottom images in Fig. 3), 21 images were used that were distorted in two channels simultaneously (either in $l$ and $\alpha, l$ and $\beta$, or $\alpha$ and $\beta$ ). The subjects made rankings for these 21 images (mixed up). Although this was a harder task than in experiments 1 and 2, it could still be performed with satisfaction. We obtained the objective rankings as follows. First, we computed the color quality metric $Q_{\text {color }}$ (Eq. 10) for different values of the weighting factors $w_{l}, w_{\alpha}$ and $w_{\beta}$. The values at which the Spearman rank-order correlation coefficient (between objective and average subjective ranking) was at maximum are respectively $w_{l}=3.3, w_{\alpha}=1.3$ and $w_{\beta}=0.9$ for the Parrot image, and $w_{l}=2.8, w_{\alpha}=0.9$ and $w_{\beta}=0.8$ for the Mandrill image. Apparently, the quality metric for $l$ dominates the color quality metric.

The coefficient $\mathrm{T}_{\mathrm{c}}$, which can take on values between 0 (no agreement) and 1 (identical rankings), was in the range of $0.96-0.99,0.83-0.90$, and $0.83-0.85$ for respectively the $l, \alpha$ and $\beta$ channels. This indicates significant agreement, at the $1 \%$ level, between the observer rankings and the ranking induced by the metric. To test the hypothesis that this observed agreement in rankings exceeds what one would expect if the rankings had been made randomly, we computed the $\mathrm{z}$ statistic. Since the probability of obtaining a $\mathrm{z}$ value greater than the computed values is $\mathrm{p}<<0.00001$ we may conclude with a high degree of confidence that the raters as a group show strong agreement with the metric ranking. After establishing this fact in the first experiment, we therefore decided to use less subjects in the rest of the experiments.

\section{CONCLUDING REMARKS}

The objective image quality measure presented in this paper shows strong correlation with human visual perception. Hence, it provides a meaningful objective measure of overall image quality, minimizing the need for time-consuming and intricate subjective tests in many digital color image processing applications.

The relative image quality ranking technique used in the present study forces the observers to collapse the ensemble of local quality variations over the entire image plane into a single judgment. Observers may use different individual criteria to weigh the relative importance of image distortions. These criteria may depend on the type of distortion and on the composition of the local image region. We may gain insight into the way these factors affect the overall perceived image quality by giving the observer a clear instruction on how the judgments should be made. For example, the observer can be instructed to judge image quality based on the worst artifact. Or, alternatively, to only judge the quality of smooth image regions, or the quality of edge regions, etc. When artifacts are suprathreshold, such instructions will change the observer's ratings. Consequently, the instructions given to the observer should reflect the technique used in the quality metric to sum errors across space and assign a quality rating to the image. Another way of handling this issue is to divide the image into small subregions and have the user specify image quality for these different image regions ${ }^{32}$. For some observers the quality of the whole image is quite as important as the quality of each image element; whereas for others the color appearance of the background elements is more relevant than the color appearance of the object elements $^{51}$. Within the scope of this paper only a small number of distortions could be investigated. Future research will focus on the perceptual attributes that prevail in the perceptual quality judgement, and the effects of their spatial distribution (image composition). 


\section{REFERENCES}

1. Gaddipatti, A., Machiraju, R. and Yagel, R., Steering image generation with wavelet based perceptual metric, EUROGRAPHICS '97, 16(3), pp. C-241-C-251, 1997.

2. Mannos, J.L. and Sakrison, D.J., The effects of a visual fidelity criterion on the encoding of images, IEEE Transactions on Information Theory, 20(4), pp. 525-536, 1974.

3. Saghri, J.A., Image quality measure based on a human visual system model, Optical Engineering, 28(7), pp. 813818, 1989.

4. Karunasekera, S.A. and Kingsbury, N.G., A distortion measure for blocking artifacts in images based on human visual sensitivity, IEEE Transactions on Image Processing, 4(6), pp. 713-724, 1995.

5. Eskicioglu, A.M. and Fisher, P.S., Image quality measures and their performance, IEEE Transactions on Communications, 43(12), pp. 2959-2965, 1995.

6. Jayant, N., Johnston, J. and Safranek, R., Signal compression based on models of human perception, Proceedings of the IEEE, 81(10), pp. 1385-1422, 1993.

7. Lubin,J. (1995). A visual discrimination model for imaging system design and evaluation. In: E.Peli (Ed.), Vision models for target detection and recognition. (pp. 245-283). Singapore: World Scientific.

8. Daly, S.J., The visible differences predictor: an algorithm for the assessment of image fidelity, In: A.B. Watson (Ed.), Digital Imaging and Human Vision, pp. 179-206, MIT Press, Cambridge, MA, 1993.

9. Watson, A.B., Hu, J., McGowan, J.F. and Mulligan, J.B., Design and performance of a digital video quality metric, In: Human Vision, Visual Processing, and Digital Display IX, SPIE-3644, pp. 168-174, The Society of PhotoOptical Instrumentation Engineers, Bellingham, WA, 1999.

10. Watson, A.B., Hu, J. and McGowan, J.F., Digital video quality metric based on human vision, Journal of Electronic Imaging, 10(1), pp. 20-29, 2001.

11. Watson, A.B., Toward a perceptual video quality metric, In: Human Vision and Electronic Imaging III, SPIE-3299, pp. 139-147, The Society of Photo-Optical Instrumentation Engineers, Bellingham,WA, 1998.

12. Teo, P.C. and Heeger, D.J., Perceptual image distortion, In: Human Vision, Visual Processing, and Digital Display $V$, SPIE-2179, pp. 127-141, The International Society for Optical Engineering, Bellingham, WA, 1994.

13. van den Branden Lambrecht, C.J., Color moving pictures quality metric, In: Proceedings of the IEEE International Conference on Image Processing, 1, pp. 885-888,1996.

14. Ahumada, A.J., Computational image quality metrics: a review, In: J. Morreale (Ed.), Society for Information Display International Symposium Digest of Technical Papers, 24, pp. 305-308, Society for Information Display, Playa del Rey, California, USA, 1993.

15. Avadhanam, N. and Algazi, V.R., Evaluation of a human-vision-system-based image fidelity metric for image compression, In: A.G. Tescher (Ed.), Applications of Digital Image Processing XXII, SPIE-3808, pp. 569-579, The International Society for Optical Engineering, Bellingham, WA, 1999.

16. Avcibas, I., Sankur, B. and Sayood, K., Statistical evaluation of image quality measure, Journal of Electronic Imaging, 11(2), pp. 206-223, 2002.

17. Bolin, M.R., Meyer, G.W. and Aase, S.O., A visual difference metric for realistic image synthesis, In: B.E. Rogowitz \& T.N. Pappas (Ed.), Human Vision and Electronic Imaging IV, SPIE-3644, pp. 106-120, The International Society for Optical Engineering, Bellingham, WA, 1999.

18. Daly, S.J., Visible differences predictor: an algorithm for the assessment of image fidelity, In: B.E. Rogowitz (Ed.), Human Vision, Visual Processing, and Digital Display III, SPIE-1666, pp. 2-15, The International Society for Optical Engineerin, Bellingham, WA, USA, 2000.

19. Farrell, J.E., Zhang, X. and van den Branden Lambrecht, C.J., Image quality metrics based on single and multichannel models of visual processing, In: Proceedings of COMPCON, pp. 1997.

20. Janssen, R., Computational image quality, The International Society for Optical Engineering, Bellingham, WA, 2001.

21. Janssen, T.J.W.M. and Blommaert, F.J.J., A computational approach to image quality, Displays, 21, pp. 129-142, 2000 . 
22. Janssen, T.J.W.M. and Blommaert, F.J.J., Information processing approach to image quality, In: B.E. Rogowitz \& T.N. Pappas (Ed.), Human Vision and Electronic Imaging V, SPIE-3959, pp. 78-87, The International Society for Optical Engineering, Bellingham, WA., USA, 2000.

23. Jung, M., Léger, D. and Gazalat, M., Univariant assessment of the quality of images, Journal of Electronic Imaging, 11(3), pp. 354-364, 2002.

24. Lai, Y.-K. and Kuo, C.-C.J., A Haar wavelet approach to compressed image quality measurement, Journal of Visual Communication and Image Representation, 11(1), pp. 17-40, 2000.

25. Lai, Y.-K., Guo, J. and Kuo, C.-C.J., Perceptual fidelity measure of digital color images, In: B.E. Rogowitz \& T.N. Pappas (Ed.), Human Vision and Electronic Imaging III, SPIE-3299, pp. 221-231, The International Society for Optical Engineering, Bellingham, WA., USA, 2002.

26. Lian, M.-S., Image evaluation using a color visual difference predictor (CVDP), In: B.E. Rogowitz \& T.N. Pappas (Ed.), Human Vision and Electronic Imaging VI, SPIE-4299, pp. 175-186, The International Society for Optical Engineering, Bellingham,WA, 2001.

27. Malo, J., Pons, A.M. and Artigas, J.M., Subjective image fidelity metric based on bit allocation of the human visual system in the DCT domain, Image and Vision Computing, 15(7), pp. 535-548, 1997.

28. van den Branden Lambrecht, C.J., A working spatio-temporal model of the human visual system for image restoration and quality assessment applications, In: Proceedings of ICASSP 96, pp. 2000.

29. Nill, N.B., A visual model weighted cosine transform for image compression and quality assessment, IEEE Transactions on Communications, 33(6), pp. 551-557, 1985.

30. Wu, H.R., van den Branden Lambrecht, C.J., Yuen, M. and Qin, B., Quantitative quality and impairment metrics for digital coded images and image sequences, In: Proceedings of the Australian Telecommunication Networks \& Applications Conference, pp. 1996.

31. Zhang, X. and Wandell, B.A., Colour image fidelity metrics evaluated using image distortion maps, Signal Processing, 70(3), pp. 201-214, 1998.

32. Fuhrmann, D.R., Baro, J.A. and Cox, J.R., Experimental evaluation of psychophysical distortion metrics for JPEGencoded images, Journal of Electronic Imaging, 4(4), pp. 397-406, 1995.

33. Fdez-Vidal, X.R., Toet, A., Garcia, J.A. and Fdez-Valdivia, J., Computing visual target distinctness through selective filtering, statistical features, and visual patterns, Optical Engineering, 39(1), pp. 267-281, 2000.

34. Garcia, J.A., Fdez-Valdivia, J., Fdez-Vidal, X.R. and Rodriguez-Sánchez, R., Computational models for predicting visual target distinctness, SPIE Optical Engineering Press, Bellingham, Washington, USA, 2001.

35. Garcia, J.A., Fdez-Valdivia, J., Fdez-Vidal, X.R. and Rodriguez-Sánchez, R., Information theoretic measure for visual target distinctness, IEEE Transactions on Pattern Analysis and Machine Intelligence PAMI, 23(4), pp. 362-383, 2001.

36. Toet, A. Computing visual target distinctness, (Report TNO-TM 1997 A-039), TNO Human Factors, Soesterberg, The Netherlands, (1997).

37. Griswold, N.C., Perceptual coding in the cosine transform domain, Optical Engineering, 19(3), pp. 306-311, 1980.

38. Jain, A.K., Image data compression: a review, Proceedings of the IEEE, 69(3), pp. 349-389, 1981.

39. Limb, J.O., Distortion criteria of the human observer, IEEE Transactions on Systems, Man and Cybernetics SMC, 9(12), pp. 778-793, 1979.

40. Lukas, F.X.J. and Budrikis, Z.L., Picture quality prediction based on a visual model, IEEE Transactions on Communications, 30(7), pp. 1679-1692, 1982.

41. Marmolin, H., Subjective MSE measures, IEEE Transactions on Systems, Man and Cybernetics SMC, 16, pp. 486489, 1986.

42. Winkler, S., Perceptual distortion metric for digital color video, In: B.E. Rogowitz \& T.N. Pappas (Ed.), Human Vision and Electronic Imaging IV, SPIE-3644, pp. 175-184, The International Society for Optical Engineering, Bellingham, WA, 1999.

43. Le Callet, P. and Barba, D., Perceptual color image quality metric using adequate error, In: B.E. Rogowitz \& T.N. Pappas (Ed.), Human Vision and Electronic Imaging VII, SPIE-4662, pp. 173-180, The International Society for Optical Engineering, Bellingham, WA., USA, 2002.

44. Zhang, X., Silverstein, D.A., Farrell, J.E. and Wandell, B.A., Color Image Quality Metric S-CIELAB and Its Application on Halftone Texture Visibility, In: pp. 2000.

45. Wang, Z. and Bovik, A.C., A universal image quality index, IEEE Signal Processing Letters, 9(3), pp. 81-84, 2002. 
46. Ruderman, D.L., Cronin, T.W. and Chiao, C.-C., Statistics of cone responses to natural images: implications for visual coding, Journal of the Optical Society of America A, 15(8), pp. 2036-2045, 1998.

47. Buchsbaum, G. and Gottschalk, A., Trichromacy, opponent colours coding and optimum colour information transmission in the retina, Proceedings of the Royal Society of London B, 220(1218), pp. 89-113, 1983.

48. Fairchild, M.D., Color appearance models, Addison Wesley Longman, Inc., Reading, MA, 1998.

49. CIE. Industrial colour difference evaluation, (Report CIE 116), International Commission on Illumination CIE, Vienna, Austria, (1995).

50. Siegel, S. and Castellan, N.J., Nonparametric statistics for the behavioral sciences. $2^{\text {nd }}$ edition, McGraw-Hill, Boston, MA, 1988.

51. Trémeau, A. and Charrier, C., Influence of chromatic changes on the perception of color image quality, Color Research and Application, 25(3), pp. 200-213, 2000. 